\title{
Preferencia de uso de un monorriel en una zona de ladera con población de bajos ingresos en la ciudad de Medellín
}

\author{
Juan Pineda-Jaramillo, M.Sc. \\ Ph.D. student, Universitat Politècnica de València, Spain. \\ Iván Sarmiento Ordosgoitia, Ph.D. \\ Professor, Department of Civil Engineering, Universidad Nacional de Colombia, Colombia.
}

\begin{abstract}
RESUMEN
La sostenibilidad es un concepto cada vez más importante en los proyectos de transporte urbano. Asimismo, las condiciones de pobreza de poblaciones en laderas de ciudades latinoamericanas requieren de soluciones novedosas y de la estimación de la demanda de viajeros. Este artículo presenta el cálculo de la demanda de un monorriel mediante modelación de elección discreta, para lo cual se parte de una investigación cualitativa a través de las técnicas de grupo focal, con el que se identifican las variables que influyen en la elección. El caso de aplicación se ubica en lo que se conoce como el Cinturón Verde Metropolitano de Medellín, en las laderas de esta ciudad. Las variables significativas para viajes cortos al centro fueron el tiempo, el costo y la comodidad, y se usaron para calibrar un Modelo Logit Mixto (MXL), y estimar la influencia que tiene cada una de ellas. El número de transbordos no resultó significativo. El conjunto de modos considerados para viajes al centro de la ciudad incluyó la caminata, el bus, un taxi informal, Metrocable, y el nuevo monorriel. Se demuestra que la probabilidad de captación del modo monorriel entre los residentes de la zona de estudio varía entre aproximadamente el 12 y $25 \%$, dependiendo de la variación de las variables influyentes en la elección modal.
\end{abstract}

PALABRAS CLAVE: Sistema de transporte limpio, monorriel, modelo de elección discreta, preferencias declaradas.

\section{INTRODUCCIÓN}

La proyección y el compromiso de generar una ciudad competitiva, incluyente, equitativa y con sentido social, promueve la concepción de planes de desarrollo urbanos capaces de contemplar y cumplir el ideal de desarrollo sostenible. Como ejemplo de este objetivo, el Plan Global de Desarrollo del Municipio de Medellín 2012-2015 tuvo entre sus programas bandera el desarrollo del cinturón verde Metropolitano. Esta es una franja que bordea el límite entre lo urbano y lo rural y que quiere ser ordenado con varias intervenciones que comprenden un camino empedrado, una cicloruta, una zona donde la vivienda se ordena evitando más dispersiones y localizaciones en zonas de riesgo, y un posible sistema limpio de movilidad de mediana capacidad.

El nuevo sistema de movilidad debe cumplir con todos los requerimientos establecidos para la incorporación de la sostenibilidad como eje principal en la toma de decisiones claves en los temas relacionados con medio ambiente, urbanismo y economía. Por lo anterior, el modo de transporte elegido es el monorriel, debido a diversos factores como la eficiencia en términos energéticos y reducción significativa de las emisiones contaminantes por persona, siendo aproximadamente 20 veces inferior a la producida por un automóvil, la reducción es de tal magnitud debido a la unión entre la eficiencia (mínimo consumo de energía eléctrica y bajo rozamiento del sistema rueda - carril) y la alta demanda (ISTAS, 2009). Además, otro de los factores de gran relevancia son la capacidad del Monorriel para adaptarse a las condiciones de 
ladera, ser seguro, silencioso, confiable, de bajos costos operativos y fácil construcción (EDU; Alcaldía de Medellín, 2 013).

Dejando a un lado la discusión de la conveniencia o no desde el punto de vista paisajístico de este sistema monorriel, el cual sigue siendo muy debatido en la ciudad, el objetivo de este artículo es identificar las variables que influyen en la elección modal para calibrar un modelo de elección discreta, MXL, y estimar la influencia que tiene cada una de estas variables en la zona nororiental de la ciudad de Medellín, con pendientes elevadas y una población caracterizada por tener bajos ingresos.

\section{ESTADO DEL ARTE}

En la región del área metropolitana de Medellín se presenta una problemática con relación a la ocupación informal del territorio, principalmente en las laderas de la ciudad, originada por migraciones de población del campo, ocasionados por la violencia de décadas pasadas y búsqueda de oportunidades de empleo. Debido a que la ocupación del territorio persistirá, es necesario aceptar esa realidad y dar una solución a este problema. Por ello se ha planteado efectuar un urbanismo de ladera y procurar llevar infraestructura apropiada, condiciones más saludables y seguras de habitabilidad (Medellin como vamos, 2014).

La solución está basada en un desarrollo urbanístico, ecológico y de transporte, que ha llevado a la propuesta de la construcción de un "cinturón verde", en el que uno de los proyectos considerados para conectar el territorio, es la construcción de un monorriel, cuyo objetivo es romper el aislamiento de la ladera, transportando a su población con un sistema de movilidad sostenible y ecológico y conectarlo con el actual sistema de transporte masivo del área metropolitana. El monorriel, con un tamaño menor que el tren pesado, atiende una capacidad de pasajeros inferior a un metro, lo cual se compensa con la inversión inicial y la contaminación visual (Barry, 2014).

Tal como se ilustra en la Fig. 1, el monorriel con $8.7 \mathrm{~km}$ tendría un impacto en cuatro comunas de la ladera nororiental de Medellín y conectaría con sistemas de movilidad actuales o propuestos así:

- El Metrocable línea $\mathrm{K}$ en el sector 1 (Santo Domingo Savio) que conecta perpendicularmente con la línea A del metro,

- Un Metrocable propuesto en el sector 2 (La Cruz) que conectaría con el Metroplús (Sistema BRT) y la línea A del Metro.

- El Metrocable línea H, en construcción durante 2015-2016 en el sector 3 (13 de Noviembre - La Sierra) que a su vez conecta con el nuevo tranvía de Ayacucho y la línea A del Metro. 


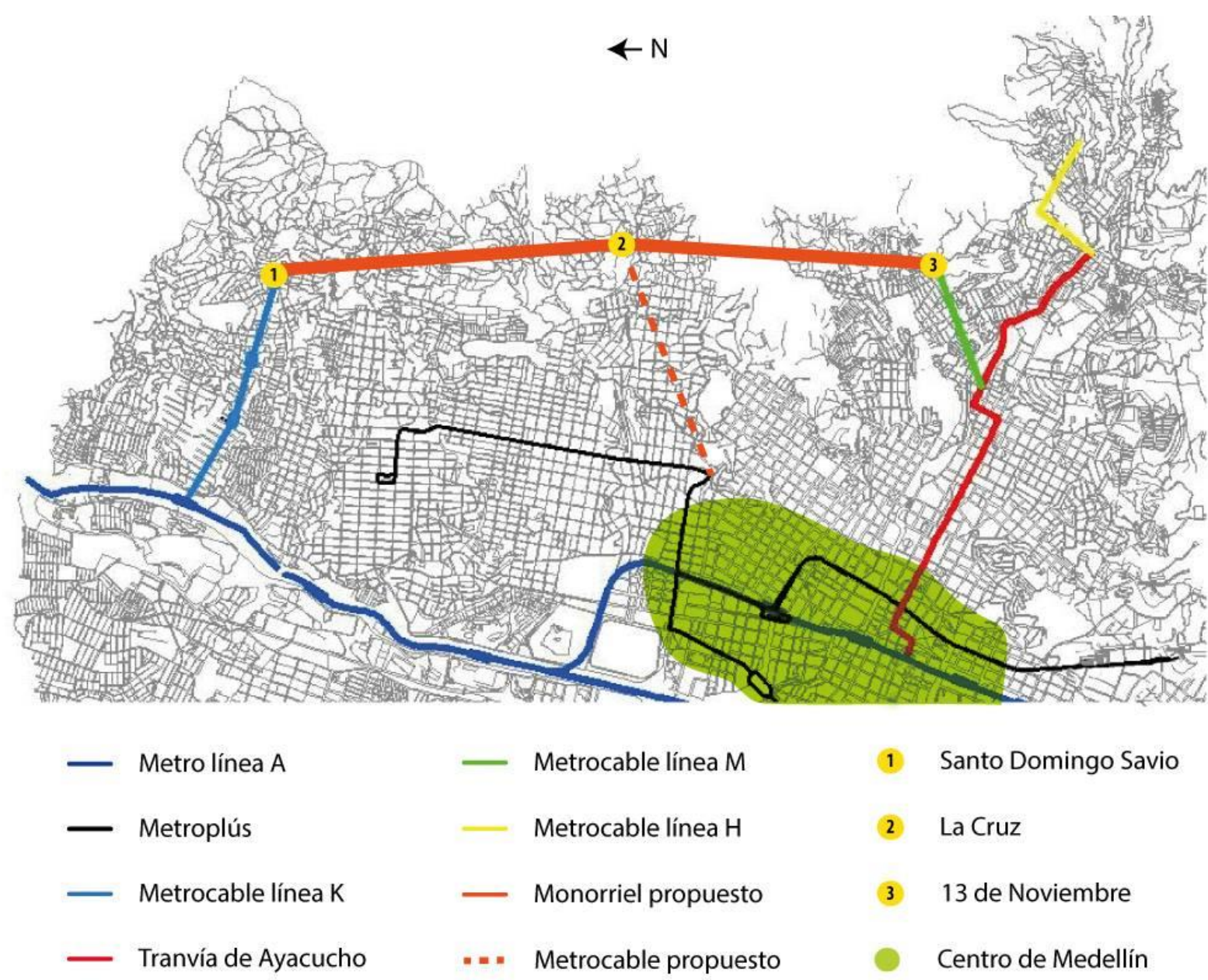

Fig. 1. Recorrido del proyecto y zona de estudio

Fuente: Elaboración propia con información de (Vicealcaldía de hábitat, movilidad, infraestructura y sostenibilidad, 2012)

Los Modelos Logit mixtos (MXL) son modelos utilizados para la determinación de preferencia de uso de modo de transporte, estos MXL son modelos más potentes que los modelos Logit Multinomial (MNL) porque consideran que los diferentes individuos tienen distintos valores tanto del tiempo como de los demás parámetros considerados. En cambio, los MNL simplifican demasiado este aspecto considerando que los individuos son un conjunto uniforme u homogéneo con la misma percepción (Ortúzar \& Willumsen, 2001).

\section{METODOLOGÍA}

La metodología para determinar la preferencia de uso de un sistema de monorriel fue:

1. Identificación de la zona de estudio, problemática de la zona, caracterización de la población de la zona y la caracterización de sus viajes.

2. Realización de grupos focales para identificar variables influyentes en la elección modal.

3. Realización de encuestas de Preferencias Declaradas (PD) y Preferencias Reveladas (PR) para el análisis de las variables influyentes en la elección modal.

4. Estimación del Modelo MXL utilizando las encuestas realizadas y el software Biogeme. 
El estudio completo comprendía un estudio de los viajes cortos al centro de la ciudad que actualmente no requieren transbordos ni pagos adicionales de tarifa, y por otro lado, los viajes largos que requieren transbordo en el centro de la ciudad para continuar hacia el norte o hacia el sur del área metropolitana. Este artículo sólo presenta la modelación de los viajes cortos al centro de la ciudad.

\section{ESTUDIO DE CASO}

\subsection{Caracterización de la zona de estudio}

La zona de estudio está ubicada al nororiente de la ciudad de Medellín, entre los barrios La Sierra y Santo Domingo Savio, con una topografía montañosa y escarpada, con pendientes de hasta el 25\%. Su población es la de las más pobres de Medellín. Esta zona será definida por tres sectores de influencia del monorriel: el Sector 1 conformado por los barrios aledaños a la estación de Metrocable en el barrio Santo Domingo Savio, el Sector 2 conformado por los barrios alrededor del barrio la Cruz y el Sector 3 conformado por barrios circundantes a la estación futura de Metrocable del barrio 13 de Noviembre (Fig. 1).

En total hay aproximadamente una población de 280 mil personas en el área de estudio, $7.5 \%$ de la población del área metropolitana de Medellín (Universidad Nacional de Colombia - Sede Medellín, 2 012).

La Tabla 1 presenta el total de viajes realizados en todos los modos en los tres sectores que se definieron en el estudio: Sector 1 de Santo Domingo (14 barrios), Sector 2 de La Cruz (9 barrios) y Sector 3 del 13 de noviembre (6 barrios), se incluyen los viajes de ida y los viajes de vuelta entre el mismo sector (viajes internos al mismo barrio) y los viajes realizados hacia y desde el centro de la ciudad en bus directo sin necesidad de realizar transbordos.

\begin{tabular}{|c|c|c|c|c|}
\hline \multirow[b]{2}{*}{ Sector } & \multirow[b]{2}{*}{ Nombre Sector } & \multicolumn{3}{|c|}{ Viajes por día } \\
\hline & & $\begin{array}{c}\text { En el mismo } \\
\text { sector }\end{array}$ & $\begin{array}{c}\text { Al centro y desde el centro de la } \\
\text { ciudad }\end{array}$ & Totales \\
\hline 1 & Santo Domingo & 1214 & 38078 & 39292 \\
\hline 2 & La Cruz & 1750 & 67250 & 69000 \\
\hline 3 & 13 de noviembre & 1118 & 42982 & 44100 \\
\hline \multicolumn{2}{|r|}{ TOTAL } & 4082 & 148310 & 152392 \\
\hline
\end{tabular}

\section{Tabla 1. Viajes realizados desde y hacia cada sector}

Fuente: Elaboración propia con información de (Universidad Nacional de Colombia - Sede Medellín, 2 012).

\subsection{Grupo focal}

Un grupo focal se define como una reunión con modalidad de entrevista grupal abierta y estructurada, en donde se procura que un grupo de individuos seleccionados por los investigadores debatan y elaboren, desde la experiencia personal, una temática que es objeto de investigación (Guía para grupos focales, 2 013). Se realizaron tres grupos focales, uno en cada sector, contando con la participación de 8 personas en cada grupo focal, donde se les realizó 5 preguntas que llevaban la discusión desde un aspecto general a lo más específico para el objetivo del grupo, algunas preguntas podían variar entre un grupo focal y otro.

De los grupos focales se identificaron cuatro variables para la elección del modo de transporte entre los residentes de los tres sectores de la zona de estudio: costo, tiempo de viaje, comodidad y número de transbordos 


\subsection{Definición de factores y diseño de la encuesta}

Para la realización de la encuesta, se realizó una discriminación entre viajes entre el mismo sector, viajes al centro de la ciudad, y viajes más largos que requerían actualmente de transbordo en el centro, para identificar el tipo de viajes que se querían modelar. En este artículo sólo se presentan los resultados para los viajes al centro. Siguiendo el diseño del experimento planteado por Kocur (Kocur, Adler, Hyman, \& Aunet, 1 982), se eligieron tres niveles de variación para las variables Tiempo de Viaje [TV], costo [C], comodidad [COM] y número de transbordos [TR]. La

\begin{tabular}{|c|c|c|c|c|c|c|c|c|c|c|c|c|}
\cline { 2 - 12 } & \multicolumn{2}{c|}{ Tiempo de Viaje } & \multicolumn{4}{c|}{ Costo } & \multicolumn{3}{c|}{ Comodidad } & \multicolumn{2}{c|}{ Número de Transbordos } \\
\cline { 2 - 12 } & 0 & 1 & 2 & 0 & 1 & 2 & 0 & 1 & 2 & 0 & 1 & 2 \\
\hline Bus & Bajo & Medio & Alto & Bajo & Medio & Alto & Bajo & Medio & Alto & Bajo & Medio & Alto \\
\hline Informal & Bajo & Medio & Alto & Bajo & Medio & Alto & Bajo & Medio & Alto & Bajo & Medio & Alto \\
\hline Metrocable & Bajo & Medio & Alto & Bajo & Medio & Alto & Bajo & Medio & Alto & Bajo & Medio & Alto \\
\hline Monorriel + Otro & Bajo & Medio & Alto & Bajo & Medio & Alto & Bajo & Medio & Alto & Bajo & Medio & Alto \\
\hline Caminata & Bajo & Medio & Alto & Bajo & Medio & Alto & Bajo & Medio & Alto & Bajo & Medio & Alto \\
\hline
\end{tabular}

Tabla 2 muestra la definición de niveles empleados en las variables definidas.

\begin{tabular}{|c|c|c|c|c|c|c|c|c|c|c|c|c|}
\cline { 2 - 12 } & \multicolumn{1}{c|}{ Tiempo de Viaje } & \multicolumn{4}{c|}{ Costo } & \multicolumn{3}{c|}{ Comodidad } & \multicolumn{2}{c|}{ Número de Transbordos } \\
\cline { 2 - 12 } & 0 & 1 & 2 & 0 & 1 & 2 & 0 & 1 & 2 & 0 & 1 & 2 \\
\hline Bus & Bajo & Medio & Alto & Bajo & Medio & Alto & Bajo & Medio & Alto & Bajo & Medio & Alto \\
\hline Informal & Bajo & Medio & Alto & Bajo & Medio & Alto & Bajo & Medio & Alto & Bajo & Medio & Alto \\
\hline Metrocable & Bajo & Medio & Alto & Bajo & Medio & Alto & Bajo & Medio & Alto & Bajo & Medio & Alto \\
\hline Monorriel + Otro & Bajo & Medio & Alto & Bajo & Medio & Alto & Bajo & Medio & Alto & Bajo & Medio & Alto \\
\hline Caminata & Bajo & Medio & Alto & Bajo & Medio & Alto & Bajo & Medio & Alto & Bajo & Medio & Alto \\
\hline
\end{tabular}

Tabla 2. Definición de niveles de variables empleadas.

Fuente: Elaboración propia.

La encuesta se diseñó en dos partes:

- La primera parte era la encuesta de Preferencias Reveladas (PR) que incluía la información relevante en el caso de la caracterización socioeconómica del individuo.

- La segunda parte consistía en la encuesta de Preferencias Declaradas (PD), con base en el número de variables y sus niveles de variación, se eligió el Plan experimental código 17a de las tablas de Kocur, lo que indica que la encuesta tendría un total de nueve casos. La encuesta PD está constituida por 9 fichas, y dentro de cada ficha se encuentra la información mostrada en el ejemplo de la Fig. 2, con base en la cual el individuo elige un modo entre los cinco posibles. 


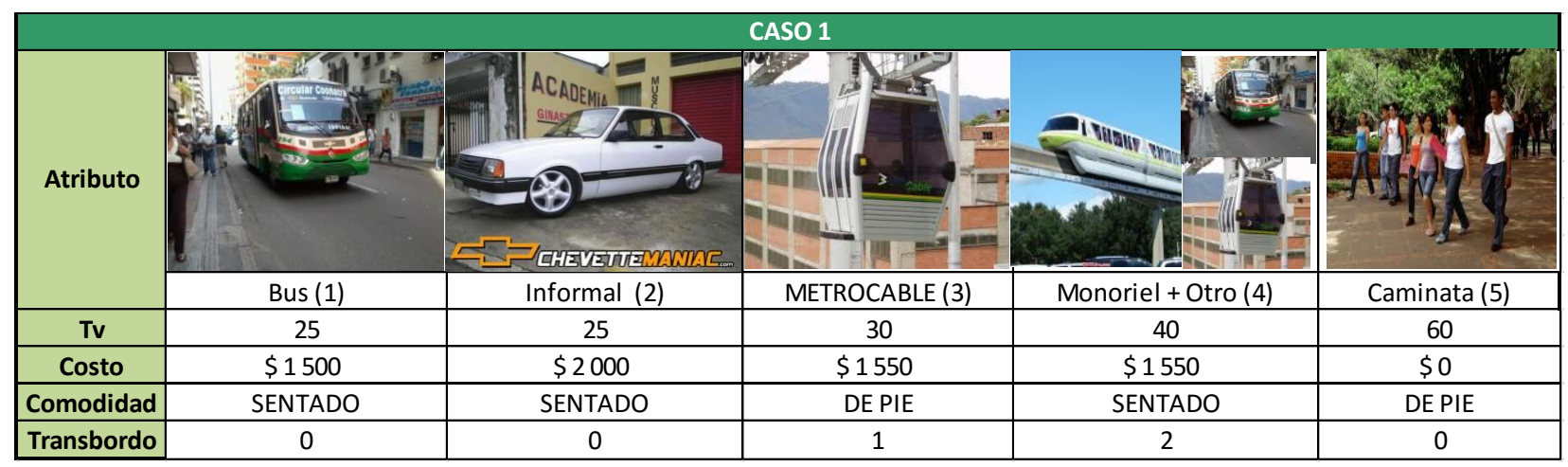

Fig. 2. Ejemplo de ficha de encuesta de PD.

Fuente: Elaboración propia.

\section{MODELO}

\subsection{Especificación y formulación del modelo}

Las variables explicativas principales utilizadas para el diseño de las preferencias declaradas y las variables secundarias recogidas en la encuesta de preferencias reveladas, fueron las siguientes:

- Tiempo de Viaje [TV].

- Costo [C].

- ComodidadZ1 (0: Sentado, 1: Otros) [COMZ1].

- ComodidadZ2 (0: Otros, 1: Apretado) [COMZ2].
- Número de Transbordos [TR]

- $\operatorname{Sexo}(1:$ Hombre, 0: Mujer) [SEX].

- Edad (0: 0-40 años, 1: Más de 40 años) [EDAD].

- Ocupación (0: Trabajo, 1: Estudio y otros) [OCU].

Entre paréntesis se muestran los rangos que fueron utilizados, para usar las variables como tipo dummy $(0,1)$.

La formulación de los modelos MXL se presenta en las siguientes ecuaciones: (1) para el bus, (2) para el taxi informal, (3) para el Metrocable, (4) para el Monorriel y (5) para la caminata:

$$
\begin{aligned}
& \boldsymbol{U}_{B U S}=\beta_{B U S}+\theta_{T V} T V_{B U S}+\theta_{C} C_{B U S}+\theta_{C O M} C O M_{B U S}+\theta_{T R} T R_{B U S} \\
& \boldsymbol{U}_{\text {INFORM }}=\beta_{\text {INFORM }}+\theta_{\text {TV }} T V_{\text {INFORM }}+\theta_{C} C_{\text {INFORM }}+\theta_{\text {COMZ1 }} \text { COMZ1 } 1_{\text {INFORM }} \\
& +\theta_{\text {COMZ2 }} \text { COMZ2 } 2_{\text {INFORM }}+\theta_{T R} T_{\text {INFORM }} \\
& \boldsymbol{U}_{\text {METROC }}=\beta_{\text {METROC }}+\theta_{\text {TV }} \text { TV } V_{\text {METROC }}+\theta_{C} C_{\text {METROC }}+\theta_{\text {COMZ1 }} \text { COMZ1 } 1_{\text {METROC }} \\
& +\theta_{\text {COMZ2 }} \text { COMZ2 }{ }_{\text {METROC }}+\theta_{T R} T R_{\text {METROC }} \\
& \boldsymbol{U}_{\text {MONOR }}=\beta_{\text {MONOR }}+\theta_{T V} T V_{\text {MONOR }}+\theta_{C} C_{\text {MONOR }}+\theta_{\text {COMZ1 }} \text { COMZ1 } 1_{\text {MONOR }} \\
& +\theta_{\text {COMZ2 }} \text { COMZ2 } 2_{\text {MONOR }}+\theta_{\text {TR }} T_{\text {MONOR }}+\theta_{v} \text { Variable } \\
& \boldsymbol{U}_{\text {CAMIN }}=\beta_{\text {CAMIN }}+\theta_{\text {TV }} T V_{\text {CAMIN }}+\theta_{C} C_{\text {CAMIN }}+\theta_{\text {COMZ1 }} \text { COMZ1 } 1_{\text {CAMIN }} \\
& +\theta_{\text {COMZ2 }} \text { COMZ2 }_{\text {CAMIN }}+\theta_{\text {TR }} T R_{\text {CAMIN }}
\end{aligned}
$$

Siendo U la utilidad de uso de cada uno de los cinco modos de transporte (bus, taxi informal, Metrocable, monorriel y caminata). La utilidad es un concepto que representa el grado de satisfacción o de insatisfacción por viajar en una determinada alternativa. Se asume por simplificación que dicha función es lineal, es decir que es la suma de lo que para cada individuo pesa cada una de las etapas de su viaje (caminar, ir dentro del vehículo, transbordar, y las condiciones del viaje tales como la comodidad, etc.). Los $\beta$ y $\theta$ corresponden a las constantes específicas y a los parámetros a estimar para cada aspecto del viaje, respectivamente (Ortúzar \& Willumsen, 2001). 


\subsection{Estimación y ranking de los modelos MXL}

Una vez especificados los modelos, se usó el software BIOGEME versión 2.0 (EPFL, 2 013), para la estimación de los parámetros, lo cual se realizó configurando distintas especificaciones sumando o restando variables al modelo para los MXL, con el objetivo de analizar los resultados de manera comparativa. La estimación del modelo MNL como del logit jerárquico (NL) no se presentan porque no daban resultados adecuados.

Para la modelación se comenzó con la especificación más compleja posible, dejando por fuera a los usuarios lexicográficos a cualquiera de los modos de transporte, involucrando todas las variables y luego se fueron formulando modelos cada vez más simples. Los usuarios lexicográficos son aquellos que se inclinan a responder siempre por lo más barato (lexicográficos del costo) o por lo más rápido sin importarles el costo (no es el caso en estos sectores de bajos ingresos) o siempre escogen el mismo modo sin importarles el costo o el tiempo (lexicográficos del modo) (Killi, Nossum, \& Veisten, 2006), Según (Carlsson \& Martinsson, 2001), las respuestas lexicográficas pueden indicar principalmente preferencias lexicográficas o una estrategia de simplificación. Estos usuarios suelen representar alrededor del 15\% de los individuos en las muestras estudiadas, y lo que se hace es excluirlos de la modelación, para tener un modelo más cercano a la racionalidad común compensatoria que es de esperar en una rutina de viaje diaria.

La Tabla 3 muestra los resultados obtenidos para un mismo modelo utilizando diferentes combinaciones de variables. Se fijó en uno la constante específica $\beta$ de la alternativa más elegida (Monorriel).

El ranking de modelos pretende seleccionar el mejor de los que están en comparación, con el fin de elegir el que por su estructura y resultados ofrezca mayor confiabilidad.

El signo de las variables el tiempo de viaje y costo es negativo, lo cual es consistente con la realidad, ya que a mayor tiempo de viaje y a mayor costo del mismo, la utilidad del usuario debe verse disminuida. El t-test indica que las variables explicativas TV, C y COM de los tres modelos son significativos al 95\% o más (t-test $\geq|1.96|)$. Se probaron varios modelos con distintas especificaciones, estos modelos difieren en el número de variables estimadas. Es interesante destacar que la variable TR (Número de transbordos) no dio significativo en ninguno de los modelos, esto puede ser debido a que como la población estudiada es de bajos ingresos, no le dan un valor muy alto a esta variable como por ejemplo a la variable costo.

\begin{tabular}{|c|c|c|c|c|c|c|c|}
\hline \multicolumn{2}{|c|}{ TIPO VIAJE } & \multicolumn{6}{c|}{ VIAJE (sin lexicográficos) } \\
\hline MODELO & \multicolumn{2}{c|}{ MXL1 } & \multicolumn{2}{c|}{ MXL2 } & \multicolumn{2}{c|}{ MXL3 } \\
\hline Variable & Parámetro & Valor & t-test & Valor & t-test & Valor & t-test \\
\hline- & $\beta 1$ & 2.36 & 0.00 & 0.0896 & 0.71 & 0.347 & 3.08 \\
\hline- & $\beta 2$ & 2.32 & 0 & 0.0522 & 0.29 & 0.287 & 1.71 \\
\hline- & $\beta 3$ & 0.529 & 0 & -0.668 & -5.73 & -0.417 & -4.13 \\
\hline- & $\beta 4$ & 0 & 0 & 0 & 0 & 0 & 0 \\
\hline Tiempo de Viaje & $\beta 5$ & -5.48 & -0.01 & -7.74 & -13.48 & -7.67 & -13.44 \\
\hline Costo & $\theta 1$ & -0.0217 & -2.339 & -0.0217 & -2.33 & -0.0217 & -2.33 \\
\hline $\begin{array}{c}\text { ComodidadZ1 (0: } \\
\text { Sentado, 1: Otros) }\end{array}$ & 03 & -0.00214 & -10.24 & -0.00214 & -10.21 & -0.00221 & -10.096 \\
\hline
\end{tabular}




\begin{tabular}{|c|c|c|c|c|c|c|c|}
\hline \multirow{2}{*}{\multicolumn{2}{|c|}{$\begin{array}{c}\text { TIPO VIAJE } \\
\text { MODELO }\end{array}$}} & \multicolumn{6}{|c|}{ VIAJE (sin lexicográficos) } \\
\hline & & \multicolumn{2}{|c|}{ MXL1 } & \multicolumn{2}{|c|}{ MXL2 } & \multicolumn{2}{|c|}{ MXL3 } \\
\hline Variable & Parámetro & Valor & t-test & Valor & t-test & Valor & $\mathrm{t}$-test \\
\hline $\begin{array}{c}\text { ComodidadZ2 (0: otros, } \\
\text { 1: Apretujado) }\end{array}$ & $\theta 4$ & -0.921 & -7.99 & -0.919 & -7.99 & -0.927 & -8.04 \\
\hline Número de transbordos & $\theta 5$ & 1.07 & 0 & & & & \\
\hline $\begin{array}{c}\text { Sexo (1: Hombre, 0: } \\
\text { Mujer) }\end{array}$ & $\theta 6$ & -0.438 & -2.59 & -0.425 & -2.51 & & \\
\hline $\begin{array}{l}\text { Edad (0: 0-40 años, 1: } \\
\text { Más de } 40 \text { años) }\end{array}$ & $\theta 7$ & -0.543 & -2.63 & -0.591 & -2.9 & & \\
\hline $\begin{array}{c}\text { Ocupación (0: Trabajo, } 1: \\
\text { Estudia y otros) }\end{array}$ & $\theta 8$ & 0.222 & 1.42 & & & & \\
\hline SIGMA1 & $\theta 92$ & -0.00139 & -8.05 & -0.00138 & -7.732 & -0.00165 & -9.29 \\
\hline Razón de verosimilitud & $\mathrm{L}(\mathrm{B})$ & \multicolumn{2}{|c|}{-1501.152} & \multicolumn{2}{|c|}{-1502.169} & \multicolumn{2}{|c|}{-1510.415} \\
\hline Rho cuadrado & $\rho^{2}$ & \multirow{2}{*}{\multicolumn{2}{|c|}{0.249}} & \multicolumn{2}{|c|}{0.249} & \multicolumn{2}{|c|}{0.240} \\
\hline Test de verosimilitud & LR & & & \multicolumn{2}{|c|}{2.034} & \multicolumn{2}{|c|}{18.526} \\
\hline
\end{tabular}

Tabla 3. Comparación de modelos según la combinación de variables elegidas.

Fuente: Elaboración propia.

Para el ranking de modelos se consideró: (a) los signos esperados de las variables costo y tiempo de viaje, (b) t-test $\geq|1.96|$ para una significancia de las variables del 95\%, (c) el test de razón de verosimilitud (LR) con $\mathrm{X}_{\mathrm{r} 95 \%}^{2} \leq 3.84$ y (d) el test estadístico $\rho^{2}$, mientras mayor es mejor. Según esto, el mejor modelo es el MXL2.

\subsection{Cuotas de mercado de los modos de transporte}

Con el modelo estimado y elegido como el mejor de todos (MXL2), se calcula la probabilidad media de uso de cada modo de transporte para varios casos. A modo de ejemplo, en la Tabla 4 se presentan las variables que se tuvieron en cuenta para calcular la probabilidad de elección de cada modo de transporte, y con base en estos valores, la Fig. 3 muestra la curva de la probabilidad del monorriel variando los costos.

\begin{tabular}{|c|c|c|c|}
\hline Modo & Costo [COP] & Tiempo [min] & Comodidad \\
\hline Bus & Variable (1550 y 1700) & 20 & Apretado \\
\hline Informal & 1700 & 20 & Apretado \\
\hline Metrocable & 1550 & 25 & Apretado \\
\hline Monorriel & Variable (1550 y 1900) & 30 & Apretado \\
\hline Caminata & 0 & 50 & Parado \\
\hline \multicolumn{3}{|c|}{ Otras variables } \\
\hline SEX & 0 (Mujer $)$ \\
\hline & EDAD & (0 a 40 años) \\
\hline
\end{tabular}

Tabla 4. Variables tenidas en cuenta para el cálculo de las probabilidades.

Fuente: Elaboración propia. 


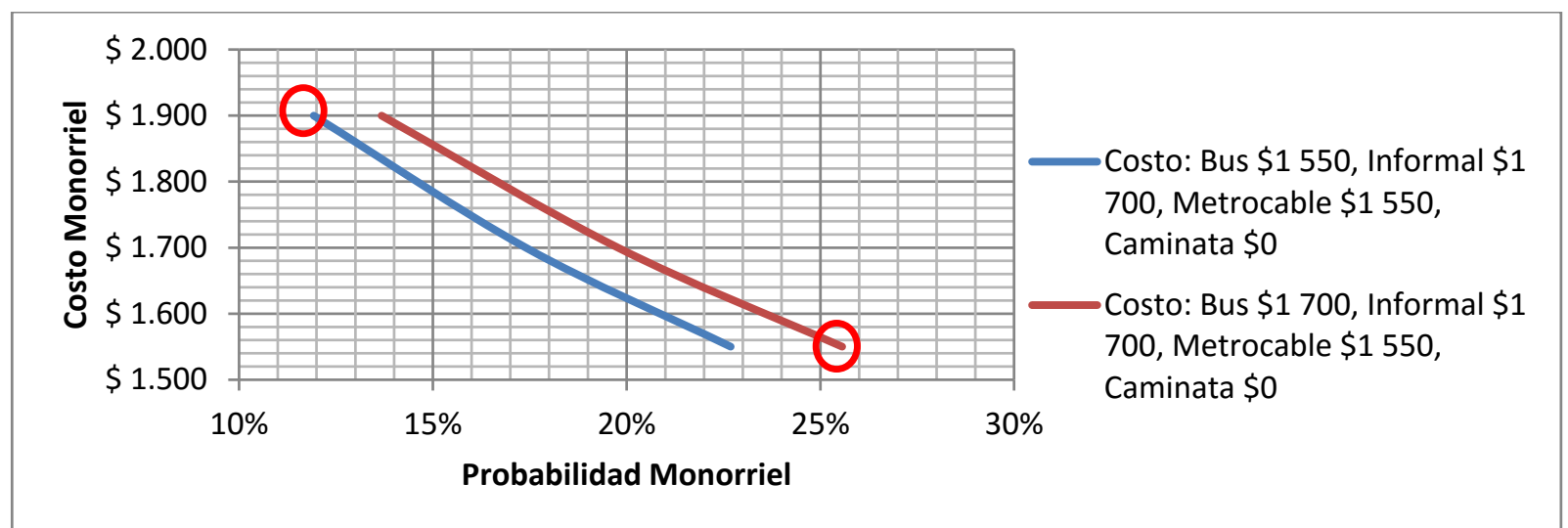

Fig. 3. Probabilidad de elección de monorriel teniendo en cuenta las variables mencionadas.

Fuente: Elaboración propia.

Con base en estos resultados, el Monorriel podría captar entre el 12\% (18 287 viajes) y el 25\% (38 098 viajes) del total de viajes que se realizan al centro de la ciudad en el sector estudiado (152 392 viajes).

\section{CONCLUSIONES}

La creación de proyectos con modos de transporte limpio debe ser estratégica, con visión a futuro y centrada en la integración social y el medio ambiente, lo que podría orientar el desarrollo en un marco de sostenibilidad, capaz de generar equidad social y el equilibrio entre el hombre y el medio ambiente.

Se ha desarrollado un modelo de elección discreta para estimar la demanda de un sistema limpio tipo monorriel en las laderas de Medellín, zona de bajos ingresos en la ciudad y con dificultades topográficas que dificultan el desarrollo de una estructura de malla vial. De los distintos modelos se puede concluir que las variables influyentes por su significancia en la elección de un modo de transporte en la zona estudiada de Medellín son, el tiempo de viaje, el costo y la comodidad. La variable transbordo no resultó significativa debido al bajo valor del tiempo de los usuarios del área de influencia del proyecto.

Con el modelo seleccionado, la probabilidad de elección del monorriel, con las variables especificadas, está entre el $12 \%$ y el $25 \%$ del total de viajes que se realizan al centro de la ciudad en el sector estudiado. Lo anterior demuestra una gran aceptación del monorriel en esta zona de la ciudad que por sus bajos ingresos y el poco desarrollo urbanístico suelen ser zonas en las que las personas se sienten olvidadas por el estado y en las que este tipo de proyecto representa una reivindicación histórica, como ya ocurrió con los Metrocables entre 2004 y 2008 en otras zonas de bajos ingresos de la ciudad.

\section{BIBLIOGRAFÍA}

Barry, K. (21 de Enero de 2014). The monorail gets a second life in Sao Paulo. Recuperado el 15 de Agosto de 2014, de http://www.wired.com/2014/01/sao-paulo-monorail/\#

Carlsson, F., \& Martinsson, P. (2001). Do hypothetical and actual marginal willingness to pay differ in choice experiments? Application to the valuation of the environment. Journal of Environmental Economics and Management, 41, 179-192. 
EDU; Alcaldía de Medellín. (2 013). Monorriel: sistema de movilidad limpia. Medellín: Alcaldía de Medellín.

EPFL. (7 de Noviembre de 2 013). BIOGEME. Obtenido de http://biogeme.epfl.ch

Guía para grupos focales, C. u. (5 de Noviembre de 2 013). Guía para grupos focales. Obtenido de http://www.slideshare.net/consultoriauniversidad/grupos-focales-2772980

ISTAS. (2009). Glosario de movilidad sostenible. Primera edición. Barcelona.

Killi, M., Nossum, A., \& Veisten, K. (2006). Lexicographic answering in travel choice: Insufficient scale extensions and steep indifference curves? European Journal of Transport and Infrastructure Research, 7, 39-62.

Kocur, G., Adler, T., Hyman, W., \& Aunet, G. (1 982). Guide to forecasting travel demand with direct utility assessment. Report No. UMTA-NH-11-0001-82. Urban Mass Transportation Administration. US Department of Transportation, Washington, DC.

Medellin como vamos. (20 de 04 de 2014). Medellin como vamos. Obtenido de Medellin como vamos: http://www.medellincomovamos.org/editoriales/cinturon-verde-metropolitanoel-buen-dios-esta-en-el-detalle

Ortúzar, J., \& Willumsen, L. G. (2001). Modelling transport. Chichester: Third Edition, John Wiley and Sons.

Universidad Nacional de Colombia - Sede Medellín. (2 012). Encuesta Origen - Destino a hogares del Valle de Aburrá. Medellín.

Vicealcaldía de hábitat, movilidad, infraestructura y sostenibilidad. (2012). Monorriel Cinturón verde. Medellín. 